\title{
Creatividad y desarrollo neuropsicológico en estudiantes de educación primaria
}

\section{Creativity and neuropsychological development in students of elementary education}

\author{
Alcides Quispe Mamani ${ }^{1 \mathrm{a}}$ \\ Universidad Peruana Unión, Lima, Perú ${ }^{1}$
}

Recibido: 12 de enero de 2020

Aceptado: 18 de noviembre de 2020

\section{Resumen}

El presente estudio ${ }^{\mathrm{b}}$ tuvo como objetivo determinar la relación entre la inteligencia creativa y la madurez neuropsicológica en estudiantes de primaria del Colegio Adventista Pedro Kalbermatter de Juliaca, 2018. La investigación fue de enfoque cuantitativo, diseño no experimental, de tipo correlacional y corte transversal. La población estuvo conformada por 168 estudiantes de primaria, de la cual se obtuvo una muestra representativa de 75 estudiantes. Los instrumentos aplicados para la recolección de datos fueron; El test de inteligencia creativa de Corbalán y Martínez (2003) y el Cuestionario de madurez neuropsicológica escolar de Portellano, Mateos y Martínez (2012). Los resultados de la presente investigación evidenciaron un valor de $(\mathrm{p}=0,047)$ de acuerdo a la Tau_b de Kendall, lo que indica según la regla de decisión planteada, aceptar la hipótesis alterna y rechazar la hipótesis nula. Se concluye en que existe correlación positiva entre las variables inteligencia creativa y madurez neuropsicológica, de igual forma entre inteligencia creativa y fluidez fonológica e inteligencia creativa y fluidez semántica. Por el contrario, no se halló correlación entre inteligencia creativa y las demás dimensiones de madurez neuropsicológicas planteadas en el presente estudio.

Palabras clave: inteligencia creativa, neuropsicología, escolaridad, educación primaria, educación

\footnotetext{
Abstract

${ }^{\mathrm{a}}$ Correspondencia al autor

E-mail: alcides.mamani@upeu.edu.pe

${ }^{\mathrm{b}} \mathrm{La}$ presente investigación se basa en la tesis de maestría del autor:

http://hdl.handle.net/20.500.12840/3129
}

The present study aimed to determine the relationship between the creative intelligence and the neurological maturity in primary students of Pedro Kalbermatter Adventist School, in Juliaca, Perú, 2018. This research was done through the quantitative approach, no experimental design, correlational and cross-sectional study. The population was one hundred sixty-eight primary students from whom was taken seventy-five students as a representative sample. The instruments used for data recollection were, the creative 
intelligence test of Corbalan and Martinez (2003) and the scholar neuropsicological matureness questionary of Portellado, Mateos and Martinez (2012). The results were that there is a $(\mathrm{p}=0,047)$ value according to the Tau_b of Kendall, it allows according to the suggested rule of decision to accept the alternate hypothesis and to reject the null hypothesis. As a conclusion, there exist a positive correlationship between creative intelligence and neuropsicological maturity, there exist positive correlationship between creative intelligence and phonological fluency and also between creative intelligence and semantic fluency. In other hand, does not exist correlationship between creative intelligence and the other neuropsicological dimensions suggested by the present study.

Keywords: Creative intelligence, neuropsychology, schooling, primary education, education

\section{Introducción}

La Organización de las Naciones Unidas para la Educación, la Ciencia y la Cultura (UNESCO, 2015) a través del Tercer estudio Regional Comparativo y Explicativo (TERCE) indica que el desempeño escolar ha ido mejorando en América Latina. Sin embargo, en el estudio realizado con más de 134 mil estudiantes del tercer y cuarto grado de educación primaria, se evidenció que los niveles de desempeño en promedio siguen siendo bajos en cursos básicos como lenguaje, matemática y ciencias naturales, estas podrían deberse a factores asociados como el apoyo socioeconómico familiar, apoyo de padres y la asistencia previa a la educación preescolar que es donde se recibe gran parte de la estimulación temprana.

Asimismo, la Organización para la Cooperación y el Desarrollo Económicos (OCDE, 2016) refiere que dichos niveles bajos de desempeño en educación primaria tienen consecuencias a largo plazo, pues estos tienden a hacer que los estudiantes abandonen los estudios en la etapa de la adolescencia y por ende suelen ser una amenaza para el desarrollo de un país. Por otro lado, la Evaluación Internacional de Estudiantes (PISA) informó sus resultados en el 2012, que uno de cuatro estudiantes no había alcanzado el nivel básico de conocimientos en cursos de matemática, lenguaje y ciencias naturales; es decir que en 64 países que conformar la OCDE, incluido países Latino Americanos, el 28\% de estudiantes se muestran por debajo del nivel de conocimiento básico al menos en una de las asignaturas mencionadas.

En el Perú, el Ministerio de Educación MINEDU (2013) a través de la evaluación censal del rendimiento escolar (ECE) a niños y niñas del segundo grado de educación primaria, dio a conocer que el $33 \%$ de estudiantes logró un aprendizaje satisfactorio en comprensión lectora y el 16\% en matemática, pero refieren que aún nos encontramos muy 
lejos de lo que realmente deberíamos lograr en todo el país, así mismo mencionan que según los resultados de las escuelas privadas han ido disminuyendo su rendimiento académico a diferencia de los centros educativos estatales en relación a años pasados. Solo en Arequipa, el diario El Pueblo (2017) informa que en el ranking de la Evaluación Censal de Estudiantes (ECE) del 2016 descendieron académicamente en asignaturas como matemática y comprensión lectora; sin embargo, el gerente regional de Educación de la región refiere que uno de los factores de esta desigualdad académica podría deberse al soporte intercultural, refuerzo extraescolar individualizado y planes de nivelación con los que no se cuenta en la ciudad de Arequipa. En la región de Puno, los datos no son muy distintos, pues realizando un estudio en la Asociación Educativa del Colegio Adventista Pedro Kalbermatter, se menciona que los estudiantes de segundo, tercero y cuarto, a diferencia de los de cuarto, quinto y sexto grado de primaria han presentado dificultades en su rendimiento académico desde el año 2017, viéndose reflejado en el ranking de notas los puestos bajos en jornadas académicas y de competencia con otras instituciones; refiere que dichos resultados pueden deberse a la falta de estimulación en el jardín, cambio constante de docentes y la falta de control por parte de los padres de familia (Apaza, 2017).

Por otro lado, la UNESCO (2015) a través del TERCE dieron a conocer que los factores asociados al bajo rendimiento escolar incluyen la asistencia previa al centro preescolar y las buenas prácticas docente. El preescolar llamado también jardín, provee a los niños y niñas la oportunidad de cuestionar comentarios o frases, además de que le provee un adecuado espacio de estimulación en su neurodesarrollo o madurez neuropsicológica (Saavedra, 2017). El MINEDU (2013) refiere que el rasgo principal de profesores con buenas prácticas docente es la creatividad, pues las formas de enseñanza que utilizan son novedosas y útiles para sus estudiantes. Además, Sevilla (2003) menciona que los docentes son guías de las experiencias cotidianas de sus estudiantes, en donde la investigación es un medio por el que reflexionan y exploran diferentes situaciones problemáticas y realidades del mundo, haciéndolos creativos; es decir que tanto el docente como el estudiante generan un ambiente de enseñanza creativo. Asimismo, en los últimos veinte años, la creatividad ha influenciado en los sistemas educativos el desarrollo del pensamiento, así como una mejor vida a nivel personal, social y económico (Medina et al., 2017).

La inteligencia creativa es definida por Sternberg (1985) como la capacidad para ajustarse a nuevas situaciones, usar conceptos o combinar información de modo novedoso 
(Fandiño, 2008). La creatividad propiamente dicha es definida por Guilford (1971) como la capacidad de generar diferentes alternativas partiendo de una información dada, poniendo énfasis en la variedad, cantidad y relevancia de los resultados (Esquivias, 2004). Aunque a través de la historia se haya generado una disyuntiva en cuanto al concepto de la creatividad, se tiene un consenso en cuanto a sus características de fluidez, flexibilidad, originalidad y elaboración de ideas (Almansa, 2012), lo último en mención, podría explicar hasta cierto punto que la creatividad ha influenciado en los sistemas educativos el desarrollo del pensamiento (Medina et al., 2017).

Por otro lado, la madurez neuropsicológica para Portellano, Mateos y Martínez (2000), es el grado de organización y desarrollo madurativo que le permite desenvolverse a las funciones cognitivas y conductuales de acuerdo a la edad cronológica del individuo (Urzúa et al., 2010), y la neuropsicología infantil se encarga de estudiar la relación de los procesos mentales superiores y cambios evolutivos en relación a su entorno (Portellano, 2007). Tomando en cuenta que la creatividad fomenta el desarrollo neurológico en menos o mayor grado (Rendón, 2009) y la madurez neuropsicológica implica el nivel de dicho desarrollo, nos permite pensar que pudiera existir cierta relación entre ambas variables de estudio.

La evidencia científica muestra que existe aún discrepancia en cuanto a la relación de la creatividad y el desarrollo neuropsicológico. Es así que, mientras Ayacho (2018); Ramírez (2015); y Vargas (2016) en sus diferentes investigaciones muestran resultados positivos en cuanto a la relación entre la creatividad y madurez neuropsicológica, los resultados de las investigaciones de Caballero y Fernández (2018); Castro y Chacón (2018); Ferrando et al. (2012); Krumm, Arán y Bustos, (2014) demuestran que no existe relación de manera precisa entre las variables de estudio y si se relacionan, son solo en algunos aspectos. Ante esta disyuntiva, es necesario comprender hasta qué punto se relacionan la creatividad y la madurez neuropsicológica, ya que ambas están asociadas al desarrollo anatomo fisiológico del cerebro cuyo efecto se expresa en los diferentes ámbitos de la vida incluido el desenvolvimiento escolar y su nivel de rendimiento. Por tanto, es el objetivo de la presente investigación determinar la relación entre la inteligencia creativa y la madurez neuropsicológica.

\section{Metodología}

El presente estudio corresponde al tipo de investigación descriptivo-correlacional, pues este permite describir las relaciones entre dos o más variables en un momento determinado, además brinda información de hechos tal cual se dan. Corresponde a un 
diseño no experimental, de corte transversal, puesto que no se manipulará las variables en cuestión y se recogerán los datos en un solo momento en la línea del tiempo (Hernández, Fernández \& Baptista, 2014).

La población con la que se trabajó estuvo conformada por 168 estudiantes del nivel primario del Colegio Adventista Pedro Kalbermatter, de ambos sexos y cuyas edades oscilan entre 6 a 11 años de edad, está ubicada en la Circunvalación Oeste - Parque el cholo de la ciudad de Juliaca. La técnica de muestreo que se utilizó fue no probabilística por conveniencia, que según Otzen y Manterola (2017) se aplica cuando la selección de individuos depende de ciertas características y limitaciones; Se denomina por conveniencia porque se toman en cuenta a los sujetos que aceptan formar parte del estudio. Se logró seleccionar a un total de 75 estudiantes.

Para la variable de inteligencia creativa se utilizó el CREA. Inteligencia creativa. Una medida cognitiva de la creatividad. La finalidad del instrumento es lograr la apreciación de la inteligencia creativa a través de una evaluación cognitiva de la creatividad individual según el indicador de generación de cuestiones, en el contexto teórico de búsqueda y solución de problemas. Para la variable de madurez neuropsicológica se utilizó el Cuestionario de Madurez Neuropsicológica Escolar (CUMANES) que fue elaborado por Portellano-Pérez et al. (2012). La finalidad del instrumento es valorar el desarrollo madurativo global del niño durante el periodo escolar. De manera precisa, permite realizar una evaluación del nivel de madurez y el rendimiento cognitivo en un amplio repertorio de funciones mentales, identificar los puntos fuertes y débiles del perfil neuropsicológico de cada niño. Asimismo permite la evaluación global del desarrollo neuropsicológico en niños normales o con algún tipo de trastorno, provee un índice de desarrollo neuropsicológico durante el periodo escolar, también permite tener una visión global de rendimiento neurocognitivo de cada niño, a través de la evaluación de 11 sub pruebas.

Para el análisis estadístico se recurrió al análisis de correlación de Tau-B de Kendall, pues se usa cuando una variable es de tipo ordinal, además está en la capacidad de calcular el grado de correlación entre dos o más variables. También, se recurrió a la prueba de normalidad para determinar el análisis estadístico paramétrico o no paramétrico. Para el vaciado de datos y análisis estadístico se usó el software estadístico SPSS 20. 


\section{Resultados}

\section{Análisis descriptivo}

Según la tabla 1 , se aprecia que en cuanto a la inteligencia creativa de los estudiantes del nivel primario del Colegio Adventista Pedro Kalbermatter; El 56\% se encuentran en un nivel alto; El 40\% en un nivel medio y el $4 \%$ se ubica en un nivel bajo.

\section{Tabla 1}

Nivel de inteligencia creativa

\begin{tabular}{llcc}
\hline & & Frecuencia & Porcentaje \\
\hline Niveles & Alto & 42 & 56,0 \\
& Medio & 30 & 40,0 \\
& Bajo & 3 & 4,0 \\
& Total & 75 & 100,0 \\
\hline
\end{tabular}

Fuente: Base de datos del SPSS

Según la tabla 2, se aprecia que en cuanto a la madurez neuropsicológica de los estudiantes del nivel primario de Colegio Adventista Pedro Kalbermatter; El 2.7\% se encuentran en un nivel alto; El 52\% en un nivel medio y el $45.3 \%$ se ubica en un nivel bajo.

Tabla 2

Nivel de madurez neuropsicológica

\begin{tabular}{llcc}
\hline & & Frecuencia & Porcentaje \\
\hline Niveles & Alto & 2 & 2,7 \\
& Medio & 39 & 52,0 \\
& Bajo & 34 & 45,3 \\
& Total & 75 & 100,0 \\
\hline
\end{tabular}

Fuente: Base de datos del SPSS

\section{Análisis de prueba de normalidad}

En la tabla 3 se aprecian los resultados de las pruebas de bondad de ajuste de Kolmogorov-Smirnov (K-S). Los datos correspondientes a la tabla indican un valor significativo en una de las variables, por lo tanto, para los análisis de correlación se recurrió a la estadística no paramétrica. 
Tabla 3

Prueba de normalidad de Kolmogorov-Smirnov

\begin{tabular}{llcc}
\hline & & $\begin{array}{c}\text { Inteligencia } \\
\text { Creativa }\end{array}$ & $\begin{array}{c}\text { Madurez } \\
\text { Neuropsicológica }\end{array}$ \\
\hline $\mathrm{N}$ & & 75 & 75 \\
Parámetros normales & & $69, \mathrm{~b}$ & 87,59 \\
& Media & 69,01 & 13,390 \\
& Desviación estándar & 19,846 &, 092 \\
Máximas diferencias & Absoluta &, 179 &, 092 \\
extremas & Positivo &, 105 &,- 060 \\
& Negativo &,- 179 &, 092 \\
Estadístico de prueba & &, 179 &, $187^{\mathrm{c}}$ \\
\hline Sig. asintótica (bilateral) & &, $000^{\mathrm{c}}$ & \\
\hline
\end{tabular}

Fuente: Base de datos del SPSS

En la tabla 3 se puede ver que los valores de significancia son 0,000 en la variable inteligencia creativa y 0,187 en la variable madurez neuropsicológica, puesto que una de las variables es menor a 0,05, nos inclinamos a rechazar la hipótesis nula. En ese sentido, podemos afirmar que los datos obtenidos no cumplen una distribución normal. Por tal motivo, los análisis estadísticos de correlación utilizados fueron no paramétricos. Para comprobar o refutar dicha correlación entre las variables, se usó la prueba Tau_b de Kendall.

\section{Análisis de correlación de prueba de hipótesis}

En relación al objetivo general

Según la tabla 4, el análisis correlacional de Tau_b de Kendall evidencia un coeficiente de correlación de valor 0,165 , sin embargo, muestra un valor $\mathrm{p}=0,047$ indicando que existe correlación entre las variables de estudio.

Tabla 4

Correlación entre inteligencia creativa y madurez neuropsicológica

\begin{tabular}{|c|c|c|c|c|}
\hline & & & $\begin{array}{c}\text { Inteligencia } \\
\text { Creativa }\end{array}$ & $\begin{array}{c}\text { Madurez } \\
\text { Neuropsicológica }\end{array}$ \\
\hline \multirow[t]{6}{*}{$\begin{array}{l}\text { tau_b de } \\
\text { Kendall }\end{array}$} & \multirow[t]{3}{*}{$\begin{array}{l}\text { Inteligencia } \\
\text { Creativa }\end{array}$} & $\begin{array}{l}\text { Coeficiente de } \\
\text { correlación }\end{array}$ & 1,000 &, $165^{*}$ \\
\hline & & Sig. (bilateral) & & ,047 \\
\hline & & $\mathrm{N}$ & 75 & 75 \\
\hline & \multirow[t]{3}{*}{$\begin{array}{l}\text { Madurez } \\
\text { Neuropsicológica }\end{array}$} & $\begin{array}{l}\text { Coeficiente de } \\
\text { correlación }\end{array}$ &, $165^{*}$ & \multirow[t]{2}{*}{1,000} \\
\hline & & Sig. (bilateral) & ,047 & \\
\hline & & $\mathrm{N}$ & 75 & 75 \\
\hline
\end{tabular}

Fuente: Base de datos del SPSS 
A un nivel de significancia del 5\%, se ha calculado el valor de $\mathrm{p}=0,047$, que indican estar dentro del margen de error planteado y un coeficiente de $r=0,165$, evidenciando correlación positiva baja entre las variables de estudio. Por tal motivo, se acepta la hipótesis alterna $\left(\mathrm{H}_{\mathrm{a}}\right)$ y se rechaza la hipótesis nula $\left(\mathrm{H}_{\mathrm{o}}\right)$ afirmando que existe relación entre la Inteligencia creativa y Madurez neuropsicológica en estudiantes de primaria del Colegio Adventista Pedro Kalbermatter de Juliaca, 2018.

En relación a los objetivos específicos

Según la tabla 5, el análisis correlacional de Tau_b de Kendall evidencia un $p$ valor menor al 0,05 en las correlaciones de Inteligencia creativa y fluidez fonológica e Inteligencia creativa y fluidez semántica, indicando que existe correlación entre las variables en mención. Por otro lado, se observa en todos los demás análisis de correlación un valor de $p$ mayor al 0,05 , lo que da a entender que no existe correlación entre dichas variables de estudio.

\section{Tabla 5}

Correlación entre inteligencia creativa y dimensiones de madurez neuropsicológica

\begin{tabular}{|c|c|c|c|}
\hline \multirow[t]{2}{*}{ Madurez Neuropsicológica } & \multicolumn{2}{|c|}{ Inteligencia Creativa } & \multirow[b]{2}{*}{ Decisión } \\
\hline & Sig. (bilateral) & $r$ & \\
\hline Comprensión audioverbal & 0,242 & 0,101 & Rechazo Hi \\
\hline Comprensión de imágenes & 0,619 & 0,045 & Rechazo Hi \\
\hline Fluidez fonológica & 0,044 & 0,176 & Acepto Hi \\
\hline Fluidez semántica & 0,014 & 0,214 & Acepto Hi \\
\hline Comprensión lectora & 0,118 & 0,136 & Rechazo Hi \\
\hline Velocidad lectora & 0,803 & 0,022 & Rechazo Hi \\
\hline Escritura Audiognósica & 0,396 & 0,077 & Rechazo Hi \\
\hline Visopercepción & 0,630 & 0,042 & Rechazo Hi \\
\hline Función ejecutiva & 0,086 & 0,150 & Rechazo Hi \\
\hline Memoria verbal & 0,476 & 0,063 & Rechazo Hi \\
\hline Memoria visual & 0,600 & 0,046 & Rechazo Hi \\
\hline Ritmo & 0,200 & 0,112 & Rechazo Hi \\
\hline
\end{tabular}

\section{Discusión}

Referente al objetivo general, los hallazgos obtenidos luego del debido procesamiento de información, muestran un $\mathrm{p}=0,047$ y un $\mathrm{r}=0,165$ dando a conocer que existe correlación positiva entre las variables inteligencia creativa y madurez 
neuropsicológica en estudiantes de primaria del Colegio Adventista Pedro Kalbermatter de Juliaca, 2018. Asimismo, Ramírez (2015) en su investigación titulada "Relación entre madurez neuropsicológica y la creatividad. Programa de intervención para fomentar la creatividad a través del desarrollo neuropsicológico entre los 7 y 11 años” y Ayacho (2018) en su investigación titulada "Relación entre madurez neuropsicológica e inteligencia creativa en estudiantes de segundo y tercer grado de primaria en la Institución Educativa Privada Internacional Nuevo Horizonte, de la Ciudad de Juliaca 2018” también encontraron como resultados la existencia de relación positiva entre la inteligencia creativa y madurez neuropsicológica. Además, otras investigaciones a fin como la de Vargas (2016) en su estudio titulado "Relación entre creatividad y cociente intelectual”, Castro y Chacón (2018) en su investigación titulada "Nivel de creatividad en niños con índices de desarrollo neuropsicológico bajo" reflejaron en sus resultados relación entre sus variables de estudio.

La evidencia de resultados obtenidos en la presente investigación y en las investigaciones recopiladas como antecedentes, es corroborada por la evidencia teórica. Es así que, Portellano (2000) al referirse a la madurez neuropsicológica como el desarrollo maduracional del cerebro, que es base para el desenvolvimiento de funciones cognitivas y conductuales de acuerdo a la edad cronológica (Urzúa, et al., 2010) evidencia la necesidad de un desarrollo maduracional también para la inteligencia creativa, definida por Stemberg y Parra (2008) como la capacidad de adaptarse a situaciones nuevas, usando conceptos o información de modo novedoso; dicho proceso creativo necesita de la activación e integración funcional de diferentes áreas cerebrales (Chávez, etal., 2004) en especial de estructuras cerebrales del hemisferio derecho que tienen predominio en la creatividad, y que a su vez, necesitan la colaboración del resto del cerebro (Escobar \& Gómez, 2006). Asimismo, se destaca la activación de los lóbulos frontales que juegan un papel muy importante en el proceso creativo (Morales, 2017) dicho sea de paso, Luria da a conocer que el desarrollo pre frontral de ambos hemisferios es más notorio entre los 6 a 8 años.

Por otro lado, mientras Pidgeon et al. (2016) refiere un rol integrador de la corteza prefrontal entre la creatividad y la cognición; De Souza et al. (2014) menciona que dicho cortex se relaciona a la adaptación de comportamientos en diferentes aspectos, funciones cognitivas, sociales y afectivas. Por otro lado, y un tanto diferente a nuestros resultados, Castro y Chacón (2018) refieren que el desarrollo neuropsicológico es importante pero no imprescindible para el para la inteligencia creativa; Ferrando et al. (2012) mencionan que la creatividad y la inteligencia o perfil cognitivo se muestran como constructos independientes; asimismo, Krumm, Arán y Bustos (2014) aunque sugieren que la habilidad 
intelectual se asocia a diferentes tipos de creatividad y sus indicadores, los resultados de su investigación muestran que la creatividad y la inteligencia son constructos separados en algunos aspectos; Caballero y Fernández (2018) terminan expresando que debemos de seguir estudiando la relación de ambas variables; lo que explicaría el nivel de correlación bajo $(r=0,165)$ obtenido en el presente estudio.

\section{Conclusiones}

Primero: A un nivel de significancia del 5\%, existe relación entre la Inteligencia creativa y madurez neuropsicológica en estudiantes de primaria del Colegio Adventista Pedro Kalbermatter de Juliaca, 2018. Por lo tanto, se acepta la hipótesis alterna de investigación y se rechaza la hipótesis nula. Segundo: A un nivel de significancia del 5\%, no existe relación entre la inteligencia creativa y la madurez neuropsicológica en su dimensión comprensión audioverbal en estudiantes de primaria del Colegio Adventista Pedro Kalbermatter de Juliaca, 2018. Por lo tanto, se rechaza la hipótesis alterna de investigación. Tercero: A un nivel de significancia del 5\%, no existe relación entre la inteligencia creativa y la Madurez neuropsicológica en su dimensión comprensión de imágenes en estudiantes de primaria del Colegio Adventista Pedro Kalbermatter de Juliaca, 2018. Por lo tanto, se rechaza la hipótesis alterna de investigación.

A investigadores, tomar en consideración los datos obtenidos en la presente investigación y buscar formas innovadoras que estimulen el desarrollo neuropsicológico. Asimismo, tomar en cuenta la posibilidad de volver a investigar las variables de estudio presentadas, bajo la misma metodología a fin de refutar o corroborar los resultados hallados puesto que aún queda camino para hacer inferencia de manera más precisa. Es importante que se consideren los datos sociodemográficos al momento de recoger la información, pues como se ha evidenciado en la presente investigación, pueden influir en los resultados si no se les toma en cuenta.

\section{Referencias}

Almansa Martínez, P. (2012). Qué es el pensamiento creativo. Index de Enfermería, 21 (3), 165-168. https://doi.org/10.4321/S1132-12962012000200012

Ayacho Palma, J. L. (2018). Relación entre madurez neuropsicológica e inteligencia creativa en estudiantes de segundo y tercer grado de primaria en la Institución 
Educativa Privada Internacional Nuevo Horizonte, de la Ciudad de Juliaca 2018. [Tesis de licenciatura, Universidad Peruana Unión].

Caballero-García, Á., \& Fernández García, M. (2018). Creatividad y rendimiento académico: un estudio de caso con alumnos de $4^{\circ}$ curso de educación secundaria. Revista Iberoamericana de Educación, 78, 77-95.

Castro-Melgar, M. T., \& Chacón-Guerra, V. G. (2018). Nivel de creatividad en niños con índices de desarrollo neuropsicológico bajo [Tesis de licenciatura, Universidad de Cuenca]. http://dspace.ucuenca.edu.ec/bitstream/123456789/30221/1/Trabajo de titulación.pdf

Chávez, R. A., Graff-Guerrero, A., García-Reyna, J. C., Vaugier, V., \& Cruz-Fuentes, C. (2004). Neurobiología de la creatividad: resultados preliminares de un estudio de activación cerebral. Salud Mental, 27, 38-46. file:///C:/Users/ALCIDES/Downloads/artículo_redalyc_58232706 (2).pdf

De Souza, L. C., Guimaráes, H. C., Teixeira, A. L., Caramelli, P., Levy, R., Dubois, B., $\&$ Volle, E. (2014). Frontal lobe neurology and the creative mind. Frontiers in Psychology, 5, 761. https://doi.org/10.3389/fpsyg.2014.00761

El Pueblo. (2017). Arequipa cae en rendimiento escolar en primaria según la prueba censal. http://elpueblo.com.pe/noticia/primera/arequipa-cae-en-rendimientoescolar-en-primaria-segun-la-prueba-censal

Escobar, A., \& Gómez-González, B. (2006). Creatividad y función cerebral. Revista Mexicana de Neurociencias, 7 (5), 391-399. http://www.medigraphic.com/pdfs/revmexneu/rmn-2006/rmn065g.pdf

Esquivias-Serrano, M. T. (2004). Creatividad: Definiciones, Antecedentes Y Aportaciones. Revista Digital Universitaria, 5 (1), 2-17. http://www.revista.unam.mx/vol.5/num1/art4/art4.htm

Fandiño-Parra, Y. J. (2008). Una enseñanza e investigación inteligentes de la inteligencia para el éxito escolar y el éxito en la vida cotidiana. Revista Iberoamericana de Educación, $\quad 46 \quad(9), \quad 1$. http://dialnet.unirioja.es/servlet/dcart?info=link\&codigo=2733159\&orden=1695 78

Ferrando, M., Bermejo, R., Sainz, M., Ferrándiz, C., Prieto, M. D., \& Soto, G. (2012). Perfiles cognitivos en alumnos con baja, media y alta creatividad. Electronic Journal of Research in Educational Psychology, 10, 967-984. http://ojs.ual.es/ojs/index.php/EJREP/article/view/1545/1893 
Hernández-Sampieri, R., Fernández Collado, C., \& Baptista Lucio, P. (2014). Metodología de la investigación. Journal of Chemical Information and Modeling. Mexico D. F.: McGraw - Hill. https://doi.org/10.1017/CBO9781107415324.004

Krumm, G., Arán Filippetti, V., \& Bustos, D. (2014). Inteligencia y creatividad: correlatos entre los constructos a través de dos estudios empíricos. Universitas Psychologica, (4), 1531-1543.

Krumm, G., Filippetti, V., \& Bustos, D. (2014). Inteligencia y creatividad: Correlatos entre los constructos a través de dos estudios empíricos. Universitas Psychologica, 13 (4), 1531-1543. https://doi.org/10.11144/Javeriana.UPSY13-4.iccc

Medina, N., Velázquez, M., Alhuay-Quispe, J., \& Aguirre, F. (2017). La Creatividad en los Niños de Prescolar, un Reto de la Educación Contemporánea. REICE. Revista Iberoamericana Sobre Calidad, $15 \quad$ (2), 153-181. https://doi.org/10.15366/reice2017.15.2.008

MINEDU. (2013). Resultados ECE 2013. http://umc.minedu.gob.pe/resultados-ece2013-3/

Morales Valiente, C. (2017). La creatividad, una revisión científica. Arquitectura $Y$ Urbanismo, $35 \quad$ (2),

1815-5898. http://www.redalyc.org/html/3768/376852683005/

OCDE. (2016). Estudio de rendimiento escolar. http://www.dw.com/es/ocde-publicaestudio-de-rendimiento-escolar/a-19037188

Otzen, T., \& Manterola, C. (2017). Técnicas de muestreo sobre una población a estudio. International Journal of Morphology, 35 (1), 227-232. https://doi.org/10.4067/S0717-95022017000100037

Pidgeon, L. M., Grealy, M., Duffy, A. H. B., Hay, L., McTeague, C., Vuletic, T., \& Gilbert, S. J. (2016). Functional neuroimaging of visual creativity: a systematic review and meta-analysis. Brain and Behavior, 6 (10). https://doi.org/10.1002/brb3.540

Portellano, J. A. (2007). Neuropsicología infantil. Madrid: Editorial Síntesis, S. A. https://kupdf.com/download/neuropsicologia-infantil-jose-antonioportellano_58d5451cdc0d60862dc34695_pdf

Ramírez Villén, V. (2015). Relación entre madurez neuropsicológica y la creatividad. Programa de intervención para fomentar la creatividad a través del desarrollo neuropsicológico entre los 7 y 11 años [Tesis de maestria, Universidad Internacional de La Rioja]. https://reunir.unir.net/handle/123456789/3242 
Rendón Uribe, M. A. (2009). Creatividad y cerebro: bases neurológicas de la creatividad. Aula $, \quad 15, \quad 117-135 . \quad$ http://rca.usal.es/ revistas_trabajo/index.php/02143402/article/view/8946

Saavedra, C. (2017). Por qué es bueno que niños y niñas asistan al jardín infantil. http://www.educacion2020.cl/noticia/por-que-es-bueno-que-ninos-y-ninasasistan-al-jardin-infantil

Sevilla, J. (2003). Alumnos y Docentes Investigadores Creativos. Umbral: Revista de educación, cultura $\quad y \quad$ sociedad, $3, \quad 102-110$. http://sisbib.unmsm.edu.pe/BibVirtualdata/publicaciones/umbral/v03_n05/a10.p df

UNESCO. (2015). Estudio TERCE de la UNESCO. Santiago: Unesco-Chile. http://www.unesco.org/fileadmin/MULTIMEDIA/FIELD/Santiago/pdf/Comunic ado-de-Prensa-2a-entrega-TERCE.pdf

Urzúa, A., Ramos, M., Alday, C., \& Alquinta, A. (2010). Madurez neuropsicológica en preescolares: propiedades psicométricas del test CUMANIN. Terapia Psicologica, 28, 13-25. www.redalyc.org/pdf/785/78516730002.pdf

Vargas-Losada, H. (2016). Relación entre creatividad y coeficiente intelectual [Tesis de maestría, Universidad Internacional de La Rioja]. https://reunir.unir.net/handle/123456789/4569 